TRANSACTIONS OF THE

AMERICAN MATHEMATICAL SOCIETY

Volume 365, Number 10, October 2013, Pages 5071-5083

S 0002-9947(2013)05693-0

Article electronically published on January 4, 2013

\title{
GLEASON PARTS AND COUNTABLY GENERATED CLOSED IDEALS IN $H^{\infty}$
}

\author{
KEI JI IZUCHI AND YUKO IZUCHI
}

Abstract. It is proved that a countably generated closed ideal in $H^{\infty}$ whose common zero set is contained in the union set of nontrivial Gleason parts of $H^{\infty}$ is generated by two Carleson-Newman Blaschke products as a closed ideal.

\section{INTRODUCTION}

Let $H^{\infty}$ be the Banach algebra of bounded analytic functions on the open unit disk $\mathbb{D}$ with the supremum norm $\|\cdot\|_{\infty}$. We denote by $M\left(H^{\infty}\right)$ the maximal ideal space of $H^{\infty}$, that is, $M\left(H^{\infty}\right)$ is the family of nonzero multiplicative linear functionals on $H^{\infty}$ with the weak*-topology. For a subset $E$ of $M\left(H^{\infty}\right)$, we denote by $\bar{E}$ the closure of $E$ in $M\left(H^{\infty}\right)$. We identify a function $f$ in $H^{\infty}$ with its Gelfand transform $\widehat{f}(m)=m(f), m \in M\left(H^{\infty}\right)$, so we think of $f$ as a continuous function on $M\left(H^{\infty}\right)$. For a sequence $\left\{a_{n}\right\}_{n}$ in $\mathbb{D}$ satisfying $\sum_{n=1}^{\infty}\left(1-\left|a_{n}\right|\right)<\infty$, we have the Blaschke product

$$
b(z)=\prod_{n=1}^{\infty} \frac{-\bar{a}_{n}}{\left|a_{n}\right|} \frac{z-a_{n}}{1-\bar{a}_{n} z}, \quad z \in \mathbb{D},
$$

where if $a_{n}=0$, we consider that $-\bar{a}_{n} /\left|a_{n}\right|=1$. We call $\left\{a_{n}\right\}_{n}$ and $b(z)$ interpolating if for any bounded sequence of complex numbers $\left\{c_{n}\right\}_{n}$ there exists $f$ in $H^{\infty}$ such that $f\left(a_{n}\right)=c_{n}$ for every $n \geq 1$. In [2], Carleson gave a characterization of interpolating sequences. A Blaschke product $B$ is said to be Carleson-Newman if $B=\prod_{j=1}^{m} b_{j}$ for finitely many interpolating Blaschke products $b_{1}, b_{2}, \cdots, b_{m}$. In this case, there are many ways to give such a factorization. If $m$ is the minimal number of interpolating Blaschke products, $B$ is said to be a Carleson-Newman Blaschke product of order $m$. In the study of the structure of $H^{\infty}$, CarlesonNewman Blaschke products have played an important role (see [3, 5, 8, 11]). For Blaschke products $b_{1}$ and $b_{2}$, we write $b_{1} \prec b_{2}$ if $b_{1}$ is a subproduct of $b_{2}$.

For $x, y \in M\left(H^{\infty}\right)$, the pseudo-hyperbolic distance is defined by

$$
\rho(x, y)=\sup \left\{|f(x)|: f(y)=0, f \in H^{\infty},\|f\|_{\infty} \leq 1\right\} .
$$

A subset $E$ of $M\left(H^{\infty}\right)$ is said to be $\rho$-separated if there is $\varepsilon>0$ such that $\rho(x, y) \geq \varepsilon$ for every $x, y \in E$ with $x \neq y$. The set

$$
P(x)=\left\{y \in M\left(H^{\infty}\right): \rho(y, x)<1\right\}
$$

Received by the editors May 15, 2011 and, in revised form, August 5, 2011.

2010 Mathematics Subject Classification. Primary 30H50, 30H05; Secondary 30J10.

Key words and phrases. Gleason part, countably generated closed ideal, Carleson-Newman Blaschke product, algebra of bounded analytic functions.

The first author was partially supported by Grant-in-Aid for Scientific Research (No.21540166), Japan Society for the Promotion of Science.

(C)2013 American Mathematical Society Reverts to public domain 28 years from publication 
is called the Gleason part of $H^{\infty}$ containing $x \in M\left(H^{\infty}\right)$. If $P(x) \neq\{x\}, P(x)$ is said to be nontrivial. We denote by $G$ the union set of all nontrivial Gleason parts in $M\left(H^{\infty}\right)$. In [7] (see also [3]), Hoffman studied the structure of Gleason parts of $H^{\infty}$ extensively. For $x \in M\left(H^{\infty}\right)$, he proved that $x \in G$ if and only if there is an interpolating Blaschke product $b$ satisfying $b(x)=0$. He also proved that for an interpolating Blaschke product $b$, there exists $\varepsilon>0$ such that $\{|b|<\varepsilon\} \subset G$, where

$$
\{|b|<\varepsilon\}=\left\{x \in M\left(H^{\infty}\right):|b(x)|<\varepsilon\right\} .
$$

This fact shows that $G$ is an open subset of $M\left(H^{\infty}\right)$, and for a Carleson-Newman Blaschke product $B$ there is $\varepsilon>0$ such that $\{|B|<\varepsilon\} \subset G$. Hoffman also showed that for a nontrivial Gleason part $P(x)$ of $H^{\infty}$, there is a one-to-one, onto and continuous map $L_{x}: \mathbb{D} \rightarrow P(x)$ such that $L_{x}(0)=x$ and $f \circ L_{x} \in H^{\infty}$ for every $f \in H^{\infty}$. For $f \in H^{\infty}$, we write

$$
Z(f)=\left\{x \in M\left(H^{\infty}\right): f(x)=0\right\} .
$$

It is known that if $b$ is an interpolating Blaschke product with zeros $\left\{z_{n}\right\}_{n}$ in $\mathbb{D}$, then $Z(b)=\overline{\left\{z_{n}\right\}_{n}}, Z(b)$ is $\rho$-separated and homeomorphic to the Stone-Čech compactification of the set of natural numbers, so $Z(b)$ is a totally disconnected set (see [6, 7]). Hence if $B$ is a Carleson-Newman Blaschke product, then $Z(B)$ is also totally disconnected. Let $f \in H^{\infty}$. For $z \in \mathbb{D}$, we denote by $\operatorname{ord}(f, z)$ the order of zero of $f$ at $z$. For $x \in G \backslash \mathbb{D}$, we define $\operatorname{ord}(f, x)=\operatorname{ord}\left(f \circ L_{x}, 0\right)$. For $x \in M\left(H^{\infty}\right) \backslash G$, we put as usual $\operatorname{ord}(f, x)=\infty$ if $f(x)=0$ and $\operatorname{ord}(f, x)=0$ if $f(x) \neq 0$. Clearly, if $b$ is an interpolating Blaschke product, then $\operatorname{ord}(b, x) \leq 1$. If $b$ is a Carleson-Newman Blaschke product of order $m$, then $\operatorname{ord}(b, x) \leq m$ for every $x$.

Let $I$ be a closed ideal in $H^{\infty}$. We write

$$
Z(I)=\bigcap_{f \in I} Z(f)
$$

and

$$
\operatorname{ord}(I, x)=\inf _{f \in I} \operatorname{ord}(f, x), \quad x \in M\left(H^{\infty}\right) .
$$

For each $1 \leq j \leq \infty$ and $f \in H^{\infty}$, we put

$$
Z_{j}(f)=\left\{x \in M\left(H^{\infty}\right): \operatorname{ord}(f, x) \geq j\right\}
$$

and

$$
Z_{j}(I)=\left\{x \in M\left(H^{\infty}\right): \operatorname{ord}(I, x) \geq j\right\} .
$$

It seems very difficult to study ideal theory in $H^{\infty}$ generally (see [1]). In [4, Gorkin, Mortini and the first author proved the following two theorems for a closed ideal $I$ satisfying $Z(I) \subset G$. In this case, by Theorem 2.3 in [5], $I$ contains a Carleson-Newman Blaschke product, so $\sup _{x \in Z(I)} \operatorname{ord}(I, x)<\infty$ and $Z(I)$ is totally disconnected (see also [14]).

Theorem A. Let I be a closed ideal in $H^{\infty}$ satisfying $Z(I) \subset G$. Then $I$ coincides with the set of all $f$ in $H^{\infty}$ satisfying ord $(f, x) \geq \operatorname{ord}(I, x)$ for every $x \in Z(I)$.

This shows that if $I_{1}, I_{2}$ are closed ideals in $H^{\infty}$ such that $Z\left(I_{i}\right) \subset G$ for $i=1,2$, $Z\left(I_{1}\right)=Z\left(I_{2}\right)$ and $\operatorname{ord}\left(I_{1}, x\right)=\operatorname{ord}\left(I_{2}, x\right)$ for every $x \in Z\left(I_{1}\right)$, then we have $I_{1}=I_{2}$. 
Theorem B. Let $I$ be a closed ideal in $H^{\infty}$ satisfying $Z(I) \subset G$ and $m=$ $\sup _{x \in Z(I)} \operatorname{ord}(I, x)$. For each $1 \leq j \leq m$, let $U_{j}$ be an open subset of $M\left(H^{\infty}\right)$ satisfying $Z_{j}(I) \subset U_{j}$. Then $I$ is algebraically generated by Carleson-Newman Blaschke products $B$ of order $m$ in $I$ such that $Z_{j}(B) \subset U_{j}$ for $1 \leq j \leq m$.

The above two theorems give us a great deal of information about closed ideals $I$ satisfying $Z(I) \subset G$. In [12, 13], the authors studied closed ideals $I$ satisfying $Z(I) \subset G$ extensively.

For a sequence $\left\{f_{n}\right\}_{n}$ in $H^{\infty}$, we denote by $I\left[f_{n}: n \geq 1\right]$ the closed ideal in $H^{\infty}$ generated by functions $f_{n}, n=1,2, \cdots$; that is,

$$
I\left[f_{n}: n \geq 1\right]=\overline{\bigcup_{n=1}^{\infty} \sum_{j=1}^{n} f_{j} H^{\infty}},
$$

where the bar indicates the closure in $H^{\infty}$. The closed ideal $I\left[f_{n}: n \geq 1\right]$ is called a countably generated closed ideal in $H^{\infty}$. In this paper, we study the structure of countably generated closed ideals $I$ satisfying $Z(I) \subset G$. For a closed subset $E$ of $M\left(H^{\infty}\right)$, let $I(E)=\left\{f \in H^{\infty}: f(x)=0, x \in E\right\}$. Then $I(E)$ is a closed ideal in $H^{\infty}$ and $E \subset Z(I(E))$. For closed ideals $I_{1}, I_{2}, \cdots, I_{m}$ in $H^{\infty}$, let $\bigotimes_{i=1}^{m} I_{i}$ and $\bar{\bigotimes}_{i=1}^{m} I_{i}$ be the tensor product and the closed tensor product of $I_{1}, I_{2}, \cdots, I_{m}$, respectively. That is, $\bigotimes_{i=1}^{m} I_{i}$ is an ideal generated by functions $\prod_{i=1}^{m} f_{i}$, where $f_{i} \in I_{i}, 1 \leq i \leq m$, and $\bar{\bigotimes}_{i=1}^{m} I_{i}=\bar{\bigotimes}_{i=1}^{m} I_{i}$. In Section 2, we shall prove the following theorem.

Theorem 1.1. Let $I$ be a closed ideal in $H^{\infty}$ satisfying $Z(I) \subset G$ and $m=$ $\sup _{x \in Z(I)} \operatorname{ord}(I, x)$. Then the following conditions are equivalent.

(i) $I$ is a countably generated closed ideal.

(ii) There are compact $\rho$-separated $G_{\delta}$-subsets $E_{1}, E_{2}, \cdots, E_{m}$ of $G$ such that $I=\bar{\bigotimes}_{j=1}^{m} I\left(E_{j}\right)$.

(iii) There is a Carleson-Newman Blaschke product $B$ of order $m$ in $I$ such that $\operatorname{ord}(B, x)=\operatorname{ord}(I, x)$ for every $x \in Z(I)$, and $Z(I)$ is a $G_{\delta}$-set.

(iv) There are two Carleson-Newman Blaschke products $B_{1}, B_{2}$ in $I$ such that $I=I\left[B_{1}, B_{2}\right]$.

For a compact $\rho$-separated $G_{\delta}$-subset $E$ of $G$, there is an interpolating Blaschke product $b$ satisfying $E \subset Z(b)$, and $I(E)$ is a countably generated closed ideal. We shall show in Example 2.14 that there exist compact $\rho$-separated $G_{\delta}$-subsets $E_{1}$ and $E_{2}$ of $G$ such that $I\left(E_{1}\right) \cap I\left(E_{2}\right)$ is not countably generated. If $I$ is a countably generated closed ideal in $H^{\infty}$, then by Theorem $1.1, Z_{j}(I)$ is a $G_{\delta}$-set for every $1 \leq j \leq \infty$. But if $I$ is the closed ideal given in Example 2.14, then $Z_{2}(I)$ is not a $G_{\delta}$-set.

\section{Countably generated Closed ideals}

To prove Theorem 1.1, we need some lemmas. For a sequence $\left\{f_{n}\right\}_{n}$ in $H^{\infty}$ and $1 \leq j \leq \infty$, it is not difficult to show that

$$
Z_{j}\left(I\left[f_{n}: n \geq 1\right]\right)=\bigcap_{n=1}^{\infty} Z_{j}\left(f_{n}\right)
$$

and

$$
\operatorname{ord}\left(I\left[f_{n}: n \geq 1\right], x\right)=\inf _{n \geq 1} \operatorname{ord}\left(f_{n}, x\right), \quad x \in Z\left(I\left[f_{n}: n \geq 1\right]\right)
$$


Lemma 2.1. Let $B$ be a Carleson-Newman Blaschke product. Then $Z_{j}(B)$ is a closed $G_{\delta}$-set for every $1 \leq j<\infty$.

Proof. Let $B=\prod_{i=1}^{k} b_{i}$, where $b_{i}$ is an interpolating Blaschke product for every $1 \leq i \leq k$. Since $\operatorname{ord}\left(b_{i}, x\right) \leq 1$ for $x \in M\left(H^{\infty}\right)$, we have that $Z_{j}(B)=\emptyset$ for $j>k$. Suppose that $1 \leq j \leq k$. Put $E_{i}=Z\left(b_{i}\right)$. Then $E_{i}$ is a closed $G_{\delta}$-set. We have

$$
Z_{j}(B)=\bigcup\left\{\bigcap_{\ell=1}^{j} E_{i_{\ell}}: 1 \leq i_{1}<i_{2}<\cdots<i_{j} \leq k\right\} .
$$

Therefore $Z_{j}(B)$ is a closed $G_{\delta^{-}}$set.

Lemma 2.2. If $f \in H^{\infty}$ and $f \neq 0$, then $Z_{j}(f)$ is a closed $G_{\delta}$-set for every $1 \leq j \leq \infty$.

Proof. Let $f=B h$, where $B$ is a Blaschke product and $h \in H^{\infty}$ satisfying $|h|>0$ on $\mathbb{D}$. Then $Z_{\infty}(h)=Z(h)$ and $Z_{\infty}(h)$ is a closed $G_{\delta}$-set. By Corollary 3.1 in [9], $Z_{\infty}(B)$ is a closed $G_{\delta^{-}}$set. Then $Z_{\infty}(f)=Z_{\infty}(B) \cup Z_{\infty}(h)$ is a closed $G_{\delta^{-}}$-set. We have

$$
\begin{aligned}
Z(f) \backslash Z_{\infty}(f) & =(Z(B) \cup Z(h)) \backslash Z_{\infty}(f) \\
& =\left(Z(B) \cup Z_{\infty}(h)\right) \backslash Z_{\infty}(f)=Z(B) \backslash Z_{\infty}(f) .
\end{aligned}
$$

By Lemma 4.6 in [9], $Z(B) \backslash Z_{\infty}(f)$ is a totally disconnected set. Hence there is a sequence of open and closed subsets $\left\{E_{n}\right\}_{n}$ of $Z(B)$ such that $Z(B) \backslash Z_{\infty}(f)=$ $\bigcup_{n=1}^{\infty} E_{n}$ and $E_{n} \cap E_{k}=\emptyset$ for $n \neq k$. Let $b_{n}$ be the subproduct of $B$ with zeros $Z(B) \cap E_{n} \cap \mathbb{D}$ counting multiplicities. Since $Z(B) \cap \mathbb{D} \subset Z(B) \backslash Z_{\infty}(f)$, we have $B=\prod_{n=1}^{\infty} b_{n}$ and $Z\left(b_{n}\right)=E_{n}$ for every $n \geq 1$. We note that $b_{n}$ is a CarlesonNewman Blaschke product. For each $1 \leq j<\infty$, we have

$$
Z_{j}(f)=Z_{\infty}(f) \cup \bigcup_{n=1}^{\infty} Z_{j}\left(b_{n}\right) .
$$

By Lemma 2.1, $Z_{j}\left(b_{n}\right)$ is a closed $G_{\delta}$-set; so is $Z_{j}(f)$.

Lemma 2.3. Let $I$ be a closed ideal in $H^{\infty}$ satisfying $Z(I) \subset G$ and $m=$ $\sup _{x \in Z(I)} \operatorname{ord}(I, x)$. Then $I$ is a countably generated closed ideal if and only if $Z_{j}(I)$ is a closed $G_{\delta}$-set for every $1 \leq j \leq m$. In this case, $I$ is generated by countably many Carleson-Newman Blaschke products.

Proof. Suppose that $I=I\left[f_{n}: n \geq 1\right]$ for a sequence $\left\{f_{n}\right\}_{n}$ in $H^{\infty}$. For each $1 \leq j \leq m$, we have $Z_{j}(I)=\bigcap_{n=1}^{\infty} Z_{j}\left(f_{n}\right)$. By Lemma 2.2. $Z_{j}(I)$ is a closed $G_{\delta}$-set.

Suppose that $Z_{j}(I)$ is a closed $G_{\delta}$-set for every $1 \leq j \leq m$. For each $1 \leq j \leq m$, let $\left\{U_{j, n}\right\}_{n}$ be a sequence of open subsets of $G$ such that $Z_{j}(I)=\bigcap_{n=1}^{\infty} U_{j, n}$. By Theorem B, there is a sequence of Carleson-Newman Blaschke products $\left\{\varphi_{n}\right\}_{n}$ in $I$ such that $Z_{j}\left(\varphi_{n}\right) \subset U_{j, n}$ for every $1 \leq j \leq m$ and $n \geq 1$. Let $J=I\left[\varphi_{n}: n \geq 1\right]$. Then $J \subset I$ and $Z(I) \subset Z(J)$. We have $Z(J) \subset Z\left(\varphi_{n}\right) \subset U_{1, n}$ for every $n \geq 1$. Then $Z(J) \subset \bigcap_{n=1}^{\infty} U_{1, n}=Z_{1}(I)=Z(I)$. Hence $Z(J)=Z(I)$.

Let $x \in Z(I)$ and $\ell=\operatorname{ord}(I, x)$. Since $\varphi_{n} \in I, \ell \leq \operatorname{ord}\left(\varphi_{n}, x\right)$ for every $n \geq 1$. Since $x \notin Z_{\ell+1}(I)$, there is a positive integer $k$ such that $x \notin U_{\ell+1, k}$. Hence 
$\ell \leq \operatorname{ord}\left(\varphi_{k}, x\right) \leq \ell$. Therefore

$$
\ell=\operatorname{ord}(I, x) \leq \operatorname{ord}(J, x) \leq \operatorname{ord}\left(\varphi_{k}, x\right)=\ell .
$$

Thus we get $\operatorname{ord}(J, x)=\operatorname{ord}(I, x)$ for every $x \in Z(I)$. By Theorem A, we have $J=I$.

The following lemma follows from Theorem 3.1 in [10].

Lemma 2.4. Let $E$ be a compact $\rho$-separated subset of $G$ and $U$ be an open subset of $M\left(H^{\infty}\right)$ satisfying $E \subset U$. Then there exists an interpolating Blaschke product $b$ such that $E \subset Z(b) \subset U$.

Lemma 2.5. Let $E$ be a compact $\rho$-separated $G_{\delta}$-subset of $G$. Then $I(E)$ is a countably generated closed ideal in $H^{\infty}, E$ is a totally disconnected set, $Z(I(E))=$ $E$ and $\operatorname{ord}(I(E), x)=1$ for every $x \in E$.

Proof. By Lemma 2.4 there is an interpolating Blaschke product $b$ such that $E \subset$ $Z(b) \subset G$. Hence $\operatorname{ord}(I(E), x)=1$ for every $x \in E$. Since $Z(b)$ is a totally disconnected set, so is $E$. Let $\left\{U_{n}\right\}_{n}$ be a sequence of open subsets of $G$ satisfying $E=\bigcap_{n=1}^{\infty} U_{n}$ and $Z(b) \cap U_{n}$ be an open and closed subset of $Z(b)$ for every $n \geq 1$. Let $b_{n}$ be the subproduct of $b$ with zeros $Z(b) \cap U_{n} \cap \mathbb{D}$. Then $E \subset Z\left(b_{n}\right) \subset U_{n}$. Let $J=I\left[b_{n}: n \geq 1\right]$. Then we have $J \subset I(E)$ and

$$
E \subset Z(I(E)) \subset Z(J) \subset \bigcap_{n=1}^{\infty} U_{n}=E .
$$

Hence $Z(I(E))=Z(J)=E$. We have $\operatorname{ord}(J, x)=1$ for every $x \in E$. By Theorem A, we get $J=I(E)$.

The following lemma follows from the definition of a closed tensor product.

Lemma 2.6. Let $I_{1}, I_{2}, \cdots, I_{m}$ be countably generated closed ideals in $H^{\infty}$. Then $\bar{\bigotimes}_{j=1}^{m} I_{j}$ is a countably generated closed ideal, $Z\left(\bar{\bigotimes}_{j=1}^{m} I_{j}\right)=\bigcup_{j=1}^{m} Z\left(I_{j}\right)$ and $\operatorname{ord}\left(\bar{\bigotimes}_{j=1}^{m} I_{j}, x\right)=\sum_{j=1}^{m} \operatorname{ord}\left(I_{j}, x\right)$ for every $x \in Z\left(\bar{\bigotimes}_{j=1}^{m} I_{j}\right)$.

For closed ideals $I_{1}, I_{2}, \cdots, I_{m}$ in $H^{\infty}$ satisfying $Z\left(I_{j}\right) \subset G$ for every $1 \leq j \leq m$, in [13. Corollary 9.15] the authors proved that $\bar{\bigotimes}_{j=1}^{m} I_{j}=\bigotimes_{j=1}^{m} I_{j}$.

Lemma 2.7. Let $I$ be a closed ideal in $H^{\infty}$ satisfying $Z(I) \subset G$ and $x \in Z(I)$. Let $B$ be a Carleson-Newman Blaschke product in $I$ and $W$ be an open subset of $M\left(H^{\infty}\right)$ satisfying $x \in W$. Then there is an open subset $U$ of $M\left(H^{\infty}\right)$ satisfying that $x \in U \subset G \cap W$ and $Z(I) \cap U$ is an open and closed subset of $Z(I)$, and there is a Carleson-Newman Blaschke product $\varphi$ of order $\operatorname{ord}(I, x)$ such that $Z(\varphi) \subset U$, $\varphi \prec B$ and $\operatorname{ord}(I, y) \leq \operatorname{ord}(\varphi, y) \leq \operatorname{ord}(I, x)$ for every $y \in Z(I) \cap U$.

Proof. Since $Z(I)$ is a totally disconnected set (see [4, Theorem 2.2]), we may take a sufficiently small open subset $U$ of $M\left(H^{\infty}\right)$ such that $x \in U \subset G \cap W$ and $Z(I) \cap U$ is an open and closed subset of $Z(I)$. Since $\operatorname{ord}(I, y)$ is upper semicontinuous in $y \in Z(I)$ (see [4, Lemma 1.2]), we may assume that $\operatorname{ord}(I, y) \leq \operatorname{ord}(I, x)$ for every $y \in Z(I) \cap U$. Let

$$
I_{U}=\left\{f \in H^{\infty}: \operatorname{ord}(f, y) \geq \operatorname{ord}(I, y), y \in Z(I) \cap U\right\} .
$$

Then by Theorem A, $I_{U}$ is a closed ideal in $H^{\infty}, I \subset I_{U}, Z\left(I_{U}\right)=Z(I) \cap U$ and $\operatorname{ord}\left(I_{U}, y\right)=\operatorname{ord}(I, y)$ for every $y \in Z(I) \cap U$. By [13, Proposition 8.9], there is a 
Carleson-Newman Blaschke product $\varphi$ of order $\operatorname{ord}(I, x)$ in $I_{U}$ such that $Z(\varphi) \subset U$, $\varphi \prec B$ and $\operatorname{ord}(\varphi, x)=\operatorname{ord}\left(I_{U}, x\right)$. For each $y \in Z(I) \cap U$, we have

$$
\operatorname{ord}(I, y)=\operatorname{ord}\left(I_{U}, y\right) \leq \operatorname{ord}(\varphi, y) \leq \operatorname{ord}(I, x) .
$$

Lemma 2.8. Let $I$ be a closed ideal in $H^{\infty}$ satisfying $Z(I) \subset G$ and $m=$ $\sup _{x \in Z(I)} \operatorname{ord}(I, x)$. Let $W_{1}, W_{2}, \cdots, W_{m}$ be open subsets of $M\left(H^{\infty}\right)$ such that $Z_{j}(I) \subset W_{j}$ for every $1 \leq j \leq m$ and $W_{m} \subset W_{m-1} \subset \cdots \subset W_{1}$. Let $B$ be a Carleson-Newman Blaschke product in I. Then there is a Carleson-Newman Blaschke product $b$ such that $b \in I, b \prec B$ and ord $(b, y) \leq j$ for every $y \in$ $Z(I) \cap\left(W_{j} \backslash W_{j+1}\right)$ and $1 \leq j \leq m$, where $W_{m+1}=\emptyset$.

Proof. For each $x \in Z(I)$, since $Z(I) \subset \bigcup_{j=1}^{m}\left(W_{j} \backslash W_{j+1}\right)$ there exists $1 \leq j \leq m$ such that $x \in W_{j} \backslash W_{j+1}$. Then $\operatorname{ord}(I, x) \leq j$. By Lemma 2.7, there is an open subset $U_{x}$ of $M\left(H^{\infty}\right)$ satisfying that $x \in U_{x} \subset G \cap W_{j}$ and $Z(I) \cap U_{x}$ is an open and closed subset of $Z(I)$, and there is a Carleson-Newman Blaschke product $\varphi_{x}$ of order $\operatorname{ord}(I, x)$ such that $Z\left(\varphi_{x}\right) \subset U_{x}, \varphi_{x} \prec B$ and $\operatorname{ord}(I, y) \leq \operatorname{ord}\left(\varphi_{x}, y\right) \leq \operatorname{ord}(I, x)$ for every $y \in Z(I) \cap U_{x}$.

Since $Z(I)$ is a compact set, there is a finite set $\left\{x_{1}, x_{2}, \cdots, x_{s}\right\}$ in $Z(I)$ such that $Z(I) \subset \bigcup_{i=1}^{s} U_{x_{i}}$. Let

$$
\begin{gathered}
E_{1}=Z(I) \cap U_{x_{1}}, \quad E_{2}=\left(Z(I) \cap U_{x_{2}}\right) \backslash\left(Z(I) \cap U_{x_{1}}\right), \\
\ldots, \quad E_{s}=\left(Z(I) \cap U_{x_{s}}\right) \backslash \bigcup_{i=1}^{s-1}\left(Z(I) \cap U_{x_{i}}\right) .
\end{gathered}
$$

Then $E_{i}$ is an open and closed subset of $Z(I), E_{i} \cap E_{j}=\emptyset$ for $i \neq j$ and $\bigcup_{i=1}^{s} E_{i}=$ $Z(I)$. It may be that $x_{i} \notin E_{i}$ for some $1 \leq i \leq s$. We may take open subsets $V_{1}, V_{2}, \cdots, V_{s}$ of $M\left(H^{\infty}\right)$ satisfying that $E_{i} \subset V_{i} \subset U_{x_{i}}$ and $\bar{V}_{i} \cap \bar{V}_{j}=\emptyset$ for $i \neq j$. Let $\psi_{i}$ be the Blaschke subproduct of $\varphi_{x_{i}}$ with zeros $Z\left(\varphi_{x_{i}}\right) \cap V_{i} \cap \mathbb{D}$ counting multiplicities. Then $Z\left(\psi_{i}\right) \cap Z\left(\psi_{j}\right)=\emptyset$ for $i \neq j$ and $\operatorname{ord}\left(\psi_{i}, y\right)=\operatorname{ord}\left(\varphi_{x_{i}}, y\right)$ for every $y \in E_{i}$ and $1 \leq i \leq s$. Let $b=\prod_{i=1}^{s} \psi_{i}$. Then $b \prec B$.

Let $y \in Z(I)$. Then there is the unique $1 \leq j \leq m$ such that $y \in W_{j} \backslash W_{j+1}$. Also there is the unique $1 \leq i \leq s$ such that $y \in E_{i}$. So we have

$$
\operatorname{ord}(b, y)=\operatorname{ord}\left(\psi_{i}, y\right)=\operatorname{ord}\left(\varphi_{x_{i}}, y\right) \leq \operatorname{ord}\left(I, x_{i}\right) .
$$

Here we have two cases.

Case 1. Suppose that $x_{i} \in W_{j} \backslash W_{j+1}$. Then we have

$$
\operatorname{ord}(I, y) \leq \operatorname{ord}\left(\varphi_{x_{i}}, y\right) \leq \operatorname{ord}\left(I, x_{i}\right) \leq j .
$$

Hence $\operatorname{ord}(I, y) \leq \operatorname{ord}(b, y) \leq j$.

Case 2. Suppose that $x_{i} \in W_{k} \backslash W_{k+1}$ for some $k \neq j$. If $k<j$, then $\operatorname{ord}\left(I, x_{i}\right) \leq$ $k<j$. Hence

$$
\operatorname{ord}(I, y) \leq \operatorname{ord}\left(\varphi_{x_{i}}, y\right)=\operatorname{ord}(b, y)<j .
$$

If $k>j$, then $y \in U_{x_{i}} \subset W_{k}$. Since $y \notin W_{j+1}$ and $W_{k} \subset W_{j+1}$, we have $y \notin W_{k}$. This is a contradiction.

By the above two cases, we have $\operatorname{ord}(I, y) \leq \operatorname{ord}(b, y) \leq j$ for every $y \in Z(I) \cap$ $\left(W_{j} \backslash W_{j+1}\right)$. By Theorem A, we have $b \in I$. Thus we get the assertion. 
Lemma 2.9. Let $I$ be a countably generated closed ideal in $H^{\infty}$ satisfying $Z(I) \subset G$ and $m=\sup _{x \in Z(I)}$ ord $(I, x)$. Let $B$ be a Carleson-Newman Blaschke product in $I$. Then there is a sequence of Carleson-Newman Blaschke products $\left\{b_{n}\right\}_{n}$ such that $b_{1} \prec B, b_{n+1} \prec b_{n}, b_{n} \in I$ for every $n \geq 1$ and for each $x \in Z(I)$ there is a positive integer $n$ satisfying $\operatorname{ord}(I, x)=\operatorname{ord}\left(b_{n}, x\right)$.

Proof. By Lemma 2.3, $Z_{j}(I)$ is a closed $G_{\delta}$-set for every $1 \leq j \leq m$. For each $1 \leq$ $j \leq m$, take a sequence of open subsets $\left\{W_{j, n}\right\}_{n}$ of $M\left(H^{\infty}\right)$ such that $\bigcap_{n=1}^{\infty} W_{j, n}=$ $Z_{j}(I)$ and $W_{j, n+1} \subset W_{j, n}$ for every $n \geq 1$. Further we may assume that $W_{j+1, n} \subset$ $W_{j, n}$ for every $1 \leq j \leq m$ and $n \geq 1$, where $W_{m+1, n}=\emptyset$ for every $n \geq 1$. By Lemma 2.8, there is a Carleson-Newman Blaschke product $b_{1}$ such that $b_{1} \in I, b_{1} \prec B$ and $\operatorname{ord}\left(b_{1}, y\right) \leq j$ for every $y \in Z(I) \cap\left(W_{j, 1} \backslash W_{j+1,1}\right)$ and $1 \leq j \leq m$. By Lemma 2.8 again, there is a Carleson-Newman Blaschke product $b_{2}$ such that $b_{2} \in I, b_{2} \prec b_{1}$ and $\operatorname{ord}\left(b_{2}, y\right) \leq j$ for every $y \in Z(I) \cap\left(W_{j, 2} \backslash W_{j+1,2}\right)$ and $1 \leq j \leq m$. Inductively we may get a sequence of Carleson-Newman Blaschke products $\left\{b_{n}\right\}_{n}$ such that $b_{n} \in I, b_{n+1} \prec b_{n}$ and $\operatorname{ord}\left(b_{n}, y\right) \leq j$ for every $y \in Z(I) \cap\left(W_{j, n} \backslash W_{j+1, n}\right)$ and $1 \leq j \leq m$.

Let $x \in Z(I)$ and $t=\operatorname{ord}(I, x)$. We consider two cases separately.

Case 1. Suppose that $t<m$. Then $x \notin Z_{t+1}(I)$ and there is a positive integer $k$ such that $x \in Z(I) \cap\left(W_{t, k} \backslash W_{t+1, k}\right)$. Hence $\operatorname{ord}\left(b_{k}, x\right) \leq t$. Since $b_{k} \in I$, we have $t=\operatorname{ord}(I, x) \leq \operatorname{ord}\left(b_{k}, x\right) \leq t$. Thus we get $\operatorname{ord}(I, x)=\operatorname{ord}\left(b_{k}, x\right)$.

Case 2. Suppose that $t=m$, that is, $\operatorname{ord}(I, x)=m$. Then $x \in Z(I) \cap\left(W_{m, n} \backslash\right.$ $\left.W_{m+1, n}\right)$ for every $n \geq 1$. Hence $\operatorname{ord}\left(b_{n}, x\right) \leq m$. Since $b_{n} \in I$, we have $m \leq$ $\operatorname{ord}\left(b_{n}, x\right)$. Thus we get $\operatorname{ord}(I, x)=\operatorname{ord}\left(b_{n}, x\right)$ for every $n \geq 1$.

The following is due to Hoffman [7].

Lemma 2.10. For any interpolating Blaschke product $b$ with zeros $\left\{z_{n}\right\}_{n}$ in $\mathbb{D}$, there exists a positive number $\lambda(b)$ such that a sequence $\left\{w_{n}\right\}_{n}$ in $\mathbb{D}$ satisfying $\rho\left(w_{n}, z_{n}\right)<\lambda(b)$ is an interpolating sequence.

Lemma 2.11. Let $I$ be a closed ideal in $H^{\infty}$ and $Z(I) \subset G$. Let $B$ be a CarlesonNewman Blaschke product in I. Then there is a Carleson-Newman Blaschke product $b$ in I satisfying the following conditions.

(i) $\operatorname{ord}(b, x)=\operatorname{ord}(B, x)$ for every $x \in Z(I) \backslash \mathbb{D}$.

(ii) $\operatorname{ord}(b, z)=\operatorname{ord}(I, z)$ for every $z \in Z(I) \cap \mathbb{D}$.

(iii) $\operatorname{ord}(b, z)=1$ for every $z \in(Z(b) \backslash Z(I)) \cap \mathbb{D}$.

Proof. Let $\varphi_{1}, \varphi_{2}, \cdots, \varphi_{m}$ be interpolating Blaschke products satisfying $B=$ $\prod_{j=1}^{m} \varphi_{j}$. Let $\lambda=\min _{1 \leq j \leq m} \lambda\left(\varphi_{j}\right)$. Then $\lambda>0$. Let $\left\{z_{n}\right\}_{n}=Z(B) \cap \mathbb{D}$ and $k_{n}=\operatorname{ord}\left(B, z_{n}\right)$. Then $\sup _{n \geq 1} k_{n}<\infty$. Let $\left\{\varepsilon_{n}\right\}_{n}$ be a sequence of numbers with $0<\varepsilon_{n}<\lambda$ such that $\varepsilon_{n} \rightarrow 0$ as $n \rightarrow \infty$. We shall move the zeros of $B$ a little. Let $n$ be a positive integer. If $z_{n} \notin Z(I)$, then take $\left\{w_{n, 1}, w_{n, 2}, \cdots, w_{n, k_{n}}\right\}$ in $\mathbb{D}$ such that $\rho\left(w_{n, i}, z_{n}\right)<\varepsilon_{n}, w_{n, i} \neq w_{n, j}$ for $i \neq j$ and

$$
\left\{w_{n, 1}, w_{n, 2}, \cdots, w_{n, k_{n}}\right\} \cap\left\{z_{n}\right\}_{n}=\emptyset .
$$

If $z_{n} \in Z(I)$, put $\ell_{n}=\operatorname{ord}\left(I, z_{n}\right)$. Then take $\left\{w_{n, 1}, w_{n, 2}, \cdots, w_{n, k_{n}}\right\}$ in $\mathbb{D}$ as the following: $\rho\left(w_{n, i}, z_{n}\right)<\varepsilon_{n}$ for every $1 \leq i \leq \ell_{n}, w_{n, 1}=w_{n, 2}=\cdots=w_{n, \ell_{n}}=z_{n}$, $w_{n, i} \neq w_{n, j}$ for every $\ell_{n} \leq i<j \leq k_{n}$ and

$$
\left\{w_{n, i}: \ell_{n}+1 \leq i \leq k_{n}\right\} \cap\left\{z_{n}\right\}_{n}=\emptyset .
$$


Further, we may assume that

$$
\left\{w_{n, 1}, w_{n, 2}, \cdots, w_{n, k_{n}}\right\} \cap\left\{w_{j, 1}, w_{j, 2}, \cdots, w_{j, k_{j}}\right\}=\emptyset
$$

for every $n \neq j$ and

$$
\sum_{n=1}^{\infty} \sum_{i=1}^{k_{n}}\left(1-\left|w_{n, i}\right|\right)<\infty .
$$

Let $b$ be the Blaschke product with zeros $\left\{w_{n, i}\right\}_{n, i}$ counting multiplicities. By Lemma 2.10 $b$ is a Carleson-Newman Blaschke product. We have $\operatorname{ord}(b, x)=$ $\operatorname{ord}(B, x)$ for every $x \in Z(I) \backslash \mathbb{D}$. It is easy to see that $b$ satisfies (ii) and (iii). Since $\operatorname{ord}(I, x) \leq \operatorname{ord}(b, x)$ for every $x \in Z(I)$, by Theorem A we have $b \in I$.

Lemma 2.12. Let $B$ be a Carleson-Newman Blaschke product and $\left\{z_{n}\right\}_{n}$ be an interpolating sequence in $\mathbb{D}$. If $0<\varepsilon<1$, then

$$
\inf _{n} \sup \left\{|B(z)|: z \in \mathbb{D}, \rho\left(z, z_{n}\right)<\varepsilon\right\}>0 .
$$

Proof. To prove the assertion, suppose not. Then there exists a subsequence $\left\{n_{j}\right\}_{j}$ such that

$$
\lim _{j \rightarrow \infty} \sup \left\{|B(z)|: z \in \mathbb{D}, \rho\left(z, z_{n_{j}}\right)<\varepsilon\right\}=0 .
$$

Let $x$ be a cluster point of $\left\{z_{n_{j}}\right\}_{j}$ in $M\left(H^{\infty}\right)$. By Hoffman's work [7], it is easy to see that $B \equiv 0$ on $P(x)$, the Gleason part of $x$. By our assumption, $B \not \equiv 0$ on $P(x)$, and this is a contradiction.

Lemma 2.13. Let $B$ be a Carleson-Newman Blaschke product and $b$ be an interpolating Blaschke product. Let $E$ be a closed $G_{\delta}$-subset of $Z(b)$. Then there is an interpolating Blaschke product $\varphi$ such that $E \subset Z(\varphi)$ and $Z(B) \cap E=Z(B) \cap Z(\varphi)$.

Proof. If $Z(B) \cap E=Z(B) \cap Z(b)$, then put $\varphi=b$. Then we get the assertion. So we assume that $Z(B) \cap E \varsubsetneqq Z(B) \cap Z(b)$. By the assumptions, there is a sequence of closed subsets $\left\{K_{n}\right\}_{n}$ of $Z(b)$ such that

$$
(Z(B) \cap Z(b)) \backslash E=\bigcup_{n=1}^{\infty} K_{n}
$$

and $K_{n} \cap K_{k}=\emptyset$ for $n \neq k$. We note that

$$
\overline{\bigcup_{n=1}^{\infty} K_{n} \backslash \bigcup_{n=1}^{\infty} K_{n} \subset E .}
$$

Take a sequence of open subsets $\left\{U_{n}\right\}_{n}$ of $M\left(H^{\infty}\right)$ such that $K_{n} \subset U_{n}, \overline{U_{n}} \cap \overline{U_{k}}=\emptyset$ for $n \neq k, E \cap \bar{U}_{n}=\emptyset$ and $Z(b) \cap U_{n}$ is an open and closed subset of $Z(b)$ for every $n \geq 1$. Let $b_{n}$ be the subproduct of $b$ with zeros $\left\{z_{n, \ell}\right\}_{\ell}:=Z(b) \cap U_{n} \cap \mathbb{D}$. Then $K_{n} \subset Z\left(b_{n}\right), E \cap Z\left(b_{n}\right)=\emptyset$ for every $n \geq 1$ and $b=\prod_{n=0}^{\infty} b_{n}$ for some interpolating Blaschke product $b_{0}$. We note that

$$
(Z(B) \cap Z(b)) \backslash \bigcup_{n=1}^{\infty} Z\left(b_{n}\right) \subset E .
$$

Let $\left\{\varepsilon_{n}\right\}_{n}$ be a sequence of numbers such that $0<\varepsilon_{n}<\lambda(b)$ and $\varepsilon_{n} \rightarrow 0$ as $n \rightarrow \infty$. By Lemma 2.12, there is a sequence of positive numbers $\left\{\delta_{n}\right\}_{n}$ such that

$$
\sup \left\{|B(z)|: z \in \mathbb{D}, \rho\left(z, z_{n, \ell}\right)<\varepsilon_{n}\right\}>\delta_{n}
$$


for every $\ell \geq 1$. For each $\ell \geq 1$, take $w_{n, \ell} \in \mathbb{D}$ satisfying $\rho\left(w_{n, \ell}, z_{n, \ell}\right)<\varepsilon_{n}$ and $\left|B\left(w_{n, \ell}\right)\right|>\delta_{n}$. By Lemma 2.10. $\left\{w_{n, \ell}\right\}_{\ell}$ is an interpolating sequence for every $n \geq 1$. For each $n \geq 1$, let $\varphi_{n}$ be the interpolating Blaschke product with zeros $\left\{w_{n, \ell}\right\}_{\ell}$. Then $Z(B) \cap Z\left(\varphi_{n}\right)=\emptyset$ and $E \cap Z\left(\varphi_{n}\right)=\emptyset$ for every $n \geq 1$. Since

$$
\sup _{\ell \geq 1} \rho\left(w_{n, \ell}, z_{n, \ell}\right) \leq \varepsilon_{n} \rightarrow 0 \quad(n \rightarrow \infty)
$$

we have

$$
Z\left(\prod_{n=1}^{\infty} b_{n}\right) \backslash \bigcup_{n=1}^{\infty} Z\left(b_{n}\right)=Z\left(\prod_{n=1}^{\infty} \varphi_{n}\right) \backslash \bigcup_{n=1}^{\infty} Z\left(\varphi_{n}\right) .
$$

Put $\varphi=b_{0} \prod_{n=1}^{\infty} \varphi_{n}$. Since

$$
\sup _{n, \ell \geq 1} \rho\left(w_{n, \ell}, z_{n, \ell}\right)<\lambda(b)
$$

by Lemma $2.10 \varphi$ is an interpolating Blaschke product. Since $E \subset Z(b)$ and $E \cap Z\left(b_{n}\right)=\emptyset$ for every $n \geq 1$, we have

$$
\begin{aligned}
E & \subset Z(b) \backslash \bigcup_{n=1}^{\infty} Z\left(b_{n}\right) \\
& =\left(Z\left(b_{0}\right) \cup Z\left(\prod_{n=1}^{\infty} b_{n}\right)\right) \backslash \bigcup_{n=1}^{\infty} Z\left(b_{n}\right) \\
& =\left(Z\left(b_{0}\right) \backslash \bigcup_{n=1}^{\infty} Z\left(b_{n}\right)\right) \cup\left(Z\left(\prod_{n=1}^{\infty} \varphi_{n}\right) \backslash \bigcup_{n=1}^{\infty} Z\left(\varphi_{n}\right)\right) \\
& =Z(\varphi) \backslash \bigcup_{n=1}^{\infty} Z\left(\varphi_{n}\right) .
\end{aligned}
$$

Hence $E \subset Z(\varphi)$. Since $Z(B) \cap Z\left(\varphi_{n}\right)=\emptyset$ for every $n \geq 1$, we have

$$
\begin{aligned}
Z(B) \cap E & \subset Z(B) \cap Z(\varphi) \subset Z(B) \cap\left(Z(\varphi) \backslash \bigcup_{n=1}^{\infty} Z\left(\varphi_{n}\right)\right) \\
& =(Z(B) \cap Z(b)) \backslash \bigcup_{n=1}^{\infty} Z\left(b_{n}\right) \subset Z(B) \cap E .
\end{aligned}
$$

Hence we get $Z(B) \cap E=Z(B) \cap Z(\varphi)$.

Proof of Theorem 1.1. (i) $\Rightarrow$ (ii) By Theorem B, there is a Carleson-Newman Blaschke product $b_{1}$ of order $m$ in $I$. By Lemma 2.11, we may assume that $\operatorname{ord}\left(b_{1}, z\right)=\operatorname{ord}(I, z)$ for every $z \in Z(I) \cap \mathbb{D}$ and $\operatorname{ord}\left(b_{1}, z\right)=1$ for every $z \in$ $\left(Z\left(b_{1}\right) \backslash Z(I)\right) \cap \mathbb{D}$. By Lemma 2.9, there is a sequence of Carleson-Newman Blaschke products $\left\{b_{n}\right\}_{n}$ such that $b_{n} \in I, b_{n+1} \prec b_{n}$ for every $n \geq 1$, and for each $x \in Z(I)$ there is a positive integer $n$ satisfying $\operatorname{ord}\left(b_{n}, x\right)=\operatorname{ord}(I, x)$.

Since the order of $b_{1}$ is equal to $m$, there are interpolating Blaschke products $\varphi_{1,1}, \varphi_{2,1}, \cdots, \varphi_{m, 1}$ such that $b_{1}=\prod_{j=1}^{m} \varphi_{j, 1}$. Since $b_{n} \in I$ and $b_{n+1} \prec b_{n}$ for every $n \geq 1$, we have $\operatorname{ord}\left(b_{n}, z\right)=\operatorname{ord}(I, z)$ for $z \in Z(I) \cap \mathbb{D}$ and $\operatorname{ord}\left(b_{n}, z\right)=1$ for $z \in\left(Z\left(b_{n}\right) \backslash Z(I)\right) \cap \mathbb{D}$. Then there are the unique interpolating Blaschke products $\varphi_{1, n}, \varphi_{2, n}, \cdots, \varphi_{m, n}$ such that $b_{n}=\prod_{j=1}^{m} \varphi_{j, n}$ and $\varphi_{j, n+1} \prec \varphi_{j, n}$ for every $1 \leq j \leq m$. We note that if $z \in Z(I) \cap \mathbb{D}$ and $\varphi_{j, 1}(z)=0$, then $\varphi_{j, n}(z)=0$ for every $n \geq 1$. 
For each $1 \leq j \leq m$, let

$$
E_{j}=Z(I) \cap \bigcap_{n=1}^{\infty} Z\left(\varphi_{j, n}\right) .
$$

By Lemma 2.3, $E_{j}$ is a compact $G_{\delta}$-set. Since $\varphi_{j, n}$ is an interpolating Blaschke product, $E_{j}$ is a $\rho$-separated set. Since $b_{n} \in I$,

$$
Z(I) \subset Z\left(b_{n}\right)=\bigcup_{j=1}^{m} Z\left(\varphi_{j, n}\right),
$$

So

$$
Z(I)=\bigcup_{j=1}^{m}\left(Z(I) \cap Z\left(\varphi_{j, n}\right)\right) .
$$

We have

$$
\bigcup_{j=1}^{m} E_{j} \subset \bigcup_{j=1}^{m}\left(Z(I) \cap Z\left(\varphi_{j, n}\right)\right)=Z(I) .
$$

Suppose that $\bigcup_{j=1}^{m} E_{j} \varsubsetneqq Z(I)$ and $y \in Z(I) \backslash \bigcup_{j=1}^{m} E_{j}$. For each $1 \leq j \leq m$, since $y \notin E_{j}$ there is a positive integer $n_{j}$ such that $y \notin Z(I) \cap Z\left(\varphi_{j, n_{j}}\right)$. Let $n=\min _{1 \leq j \leq m} n_{j}$. Then

$$
Z(I) \cap Z\left(\varphi_{j, n}\right) \subset Z(I) \cap Z\left(\varphi_{j, n_{j}}\right) .
$$

Hence

$$
y \notin \bigcup_{j=1}^{m}\left(Z(I) \cap Z\left(\varphi_{j, n}\right)\right)=Z(I) .
$$

But this is a contradiction. Thus we get

$$
Z(I)=\bigcup_{j=1}^{m} E_{j}
$$

Let $x \in Z(I)$. Then there is a positive integer $n_{1}$ such that $\operatorname{ord}\left(b_{n_{1}}, x\right)=$ $\operatorname{ord}(I, x)$. We write $\ell=\operatorname{ord}(I, x)$. Then there are positive integers $j_{1}, j_{2}, \cdots, j_{\ell}$ such that

$$
\operatorname{ord}\left(\prod_{i=1}^{\ell} \varphi_{j_{i}, n_{1}}, x\right)=\ell \quad \text { and } \operatorname{ord}\left(b_{n_{1}} / \prod_{i=1}^{\ell} \varphi_{j_{i}, n_{1}}, x\right)=0 .
$$

Since $b_{n} \in I$ and $b_{n} \prec b_{n_{1}}$ for every $n \geq n_{1}, \operatorname{ord}\left(b_{n}, x\right)=\ell$ and $\varphi_{j_{i}, n}(x)=0$ for every $1 \leq i \leq \ell$ and $n \geq n_{1}$. Thus for any $n \geq n_{1}$ we have

$$
\begin{aligned}
\operatorname{ord}(I, x) & =\operatorname{ord}\left(b_{n}, x\right)=\operatorname{ord}\left(\prod_{j=1}^{m} \varphi_{j, n}, x\right) \\
& =\sum_{i=1}^{\ell} \operatorname{ord}\left(\varphi_{j_{i}, n}, x\right)=\#\left\{j: x \in E_{j}, 1 \leq j \leq m\right\},
\end{aligned}
$$

where \#A denotes the number of elements in a set $A$. Let

$$
J=\bar{\bigotimes}_{j=1}^{m} I\left(E_{j}\right)
$$


By Lemma 2.5, we have $\operatorname{ord}\left(I\left(E_{j}\right), x\right)=1$ for every $x \in E_{j}$ and $Z\left(I\left(E_{j}\right)\right)=E_{j}$ for every $1 \leq j \leq m$. Hence by Lemma 2.6, $Z(J)=\bigcup_{j=1}^{m} E_{j}=Z(I)$ and

$$
\operatorname{ord}(J, x)=\sum_{j=1}^{m} \operatorname{ord}\left(I\left(E_{j}\right), x\right)=\#\left\{j: x \in E_{j}, 1 \leq j \leq m\right\}
$$

for every $x \in Z(I)$. By Theorem A, we have $I=J=\bar{\bigotimes}_{j=1}^{m} I\left(E_{j}\right)$.

(ii) $\Rightarrow$ (iii) Suppose that condition (ii) holds. By Lemma 2.6.

$$
Z(I)=\bigcup_{j=1}^{m} Z\left(I\left(E_{j}\right)\right)=\bigcup_{j=1}^{m} E_{j}
$$

so $Z(I)$ is a $G_{\delta^{-}}$-set. By Lemma 2.4, for each $1 \leq j \leq m$ there is an interpolating Blaschke product $\varphi_{j}$ such that $E_{j} \subset Z\left(\varphi_{j}\right)$. Let $\Phi=\prod_{j=1}^{m} \varphi_{j}$. By Lemma 2.13, for each $1 \leq j \leq m$ there exists an interpolating Blaschke product $b_{j}$ such that $E_{j} \subset Z\left(b_{j}\right)$ and $Z(\Phi) \cap Z\left(b_{j}\right)=Z(\Phi) \cap E_{j}=E_{j}$. We note that $Z(I) \subset Z(\Phi)$. Let $B=\prod_{j=1}^{m} b_{j}$. Then for any $x \in Z(I)$, we have

$$
\begin{aligned}
\operatorname{ord}(B, x) & =\operatorname{ord}\left(\prod_{j=1}^{m} b_{j}, x\right)=\sum_{j=1}^{m} \operatorname{ord}\left(b_{j}, x\right) \\
& =\#\left\{j: x \in E_{j}, 1 \leq j \leq m\right\} .
\end{aligned}
$$

By Lemmas 2.5] and 2.6. we have

$$
\begin{aligned}
\operatorname{ord}(I, x) & =\operatorname{ord}\left(\bar{\bigotimes}_{j=1}^{m} I\left(E_{j}\right), x\right)=\sum_{j=1}^{m} \operatorname{ord}\left(I\left(E_{j}\right), x\right) \\
& =\#\left\{j: x \in E_{j}, 1 \leq j \leq m\right\} .
\end{aligned}
$$

Thus we get $\operatorname{ord}(B, x)=\operatorname{ord}(I, x)$ for every $x \in Z(I)$. By Theorem A, we have $B \in I$.

(iii) $\Rightarrow$ (iv) Suppose that condition (iii) holds. Let $B_{1}$ be a Carleson-Newman Blaschke product of order $m$ in $I$ satisfying $\operatorname{ord}\left(B_{1}, x\right)=\operatorname{ord}(I, x)$ for every $x \in$ $Z(I)$. Let $\varphi_{1}, \varphi_{2}, \cdots, \varphi_{m}$ be interpolating Blaschke products satisfying $B_{1}=$ $\prod_{j=1}^{m} \varphi_{j}$. For each $1 \leq j \leq m$, let $E_{j}=Z(I) \cap Z\left(\varphi_{j}\right)$. Since $Z(I)$ is a $G_{\delta^{-}}$set, $E_{j}$ is a closed $G_{\delta}$-set. By Lemma 2.13, there is an interpolating Blaschke product $b_{j}$ such that $Z\left(B_{1}\right) \cap Z\left(b_{j}\right)=E_{j}$. Let $B_{2}=\prod_{j=1}^{m} b_{j}$. For any $x \in Z(I)$, we have

$$
\begin{aligned}
\operatorname{ord}\left(B_{2}, x\right) & =\sum_{j=1}^{m} \operatorname{ord}\left(b_{j}, x\right)=\#\left\{j: x \in E_{j}, 1 \leq j \leq m\right\} \\
& =\operatorname{ord}\left(B_{1}, x\right) \geq \operatorname{ord}(I, x) .
\end{aligned}
$$

By Theorem A, we have $B_{2} \in I$. We also have

$$
\begin{aligned}
Z\left(B_{1}\right) \cap Z\left(B_{2}\right) & =Z\left(B_{1}\right) \cap \bigcup_{j=1}^{m} Z\left(b_{j}\right)=\bigcup_{j=1}^{m} E_{j} \\
& =Z(I) \cap Z\left(B_{1}\right)=Z(I) .
\end{aligned}
$$

Let $J=I\left[B_{1}, B_{2}\right]$. Then $Z(J)=Z(I)$ and $\operatorname{ord}(J, x)=\operatorname{ord}(I, x)$ for every $x \in Z(I)$. By Theorem A again, we have $J=I$.

(iv) $\Rightarrow$ (i) is trivial. 
In the following example, we shall show that there exist compact $\rho$-separated $G_{\delta}$-subsets $E_{1}$ and $E_{2}$ of $G$ such that the ideal $I\left(E_{1}\right) \cap I\left(E_{2}\right)$ is not countably generated.

Example 2.14. Let $\left\{\theta_{k}\right\}_{k}$ be a sequence of numbers such that $0<\theta_{k+1}<\theta_{k}<1$ and $\theta_{k} \rightarrow 0$ as $k \rightarrow \infty$. It is known that there is an interpolating Blaschke product $B_{1}$ with zeros $\left\{z_{n}\right\}_{n}$ in $\mathbb{D}$ such that

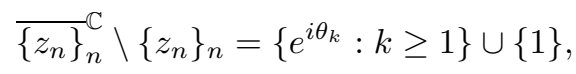

where ${\overline{\left\{z_{n}\right\}_{n}}}_{n}^{\mathbb{C}}$ is the closure of $\left\{z_{n}\right\}_{n}$ in $\mathbb{C}$. Let $\mathbb{N}$ be the set of positive integers. We may divide $\mathbb{N}$ as $\mathbb{N}=\bigcup_{k=1}^{\infty} N_{k}$ such that $N_{k} \cap N_{j}=\emptyset$ for $k \neq j$ and

$$
\overline{\left\{z_{n}: n \in N_{k}\right\}}{ }^{\mathbb{C}} \backslash\left\{z_{n}: n \in N_{k}\right\}=\left\{e^{i \theta_{k}}\right\}, \quad k \in \mathbb{N} .
$$

Let $b_{k}$ be the subproduct of $B_{1}$ with zeros $\left\{z_{n}: n \in N_{k}\right\}$. Then $B_{1}=\prod_{k=1}^{\infty} b_{k}$. Let $\left\{\varepsilon_{k}\right\}_{k}$ be a sequence of numbers such that $0<\varepsilon_{k}<1$ and $\varepsilon_{k} \rightarrow 0$ as $k \rightarrow \infty$. Let $q_{k}(z)=\left(b_{k}(z)-\varepsilon_{k}\right) /\left(1-\varepsilon_{k} b_{k}(z)\right)$. Taking smaller $\varepsilon_{k}$, we may assume that $B_{2}:=\prod_{k=1}^{\infty} q_{k}$ is an interpolating Blaschke product and

$$
\left(\bigcup_{k=1}^{\infty} Z\left(b_{k}\right)\right) \cap\left(\bigcup_{k=1}^{\infty} Z\left(q_{k}\right)\right)=\emptyset .
$$

Let

$$
E_{1}=Z\left(B_{1}\right) \backslash \mathbb{D} \quad \text { and } \quad E_{2}=Z\left(B_{2}\right) \backslash \mathbb{D} .
$$

Then $E_{1}, E_{2}$ are compact $\rho$-separated $G_{\delta}$-subsets of $G$,

$$
E_{1}=\left(\bigcup_{k=1}^{\infty}\left(Z\left(b_{k}\right) \backslash \mathbb{D}\right)\right) \cup\left(E_{1} \backslash \bigcup_{k=1}^{\infty} Z\left(b_{k}\right)\right)
$$

and

$$
E_{2}=\left(\bigcup_{k=1}^{\infty}\left(Z\left(q_{k}\right) \backslash \mathbb{D}\right)\right) \cup\left(E_{2} \backslash \bigcup_{k=1}^{\infty} Z\left(q_{k}\right)\right) .
$$

By Lemma 2.5. $I\left(E_{1}\right)$ and $I\left(E_{2}\right)$ are countably generated closed ideals in $H^{\infty}$. Let $I=I\left(E_{1}\right) \cap I\left(E_{2}\right)$. Then $I=I\left(E_{1} \cup E_{2}\right)$. By the construction, we may check that

$$
E_{1} \backslash \bigcup_{k=1}^{\infty} Z\left(b_{k}\right)=E_{2} \backslash \bigcup_{k=1}^{\infty} Z\left(q_{k}\right)
$$

and

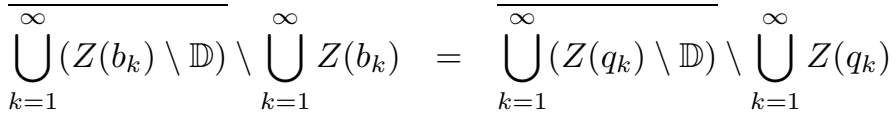

$$
\begin{aligned}
& \varsubsetneqq E_{1} \backslash \bigcup_{k=1}^{\infty} Z\left(b_{k}\right) \text {. }
\end{aligned}
$$

Let $\Omega$ be the set of all subproducts $q$ of $B_{2}$ satisfying

$$
\bigcup_{k=1}^{\infty}\left(Z\left(q_{k}\right) \backslash \mathbb{D}\right) \subset Z(q) .
$$


Then we have $B_{1} q \in I$ for every $q \in \Omega$ and

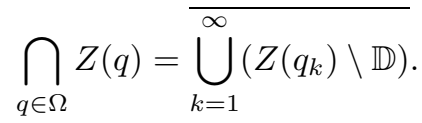

By this fact, we have

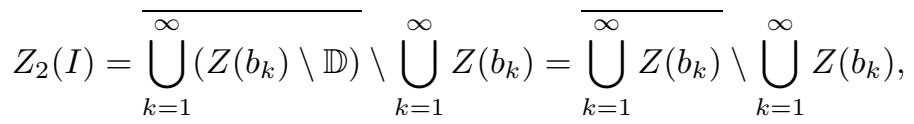

and $Z_{2}(I)$ is not a $G_{\delta}$-set (see Example 2.9 in [12]). By Lemma 2.3, $I$ is not countably generated. We note that $I=I\left(E_{1}\right) \bar{\otimes} I\left(\overline{E_{2} \backslash E_{1}}\right)$.

\section{REFERENCES}

[1] J. Bourgain, On finitely generated closed ideals in $H^{\infty}(\mathbb{D})$, Ann. Inst. Fourier (Grenoble) 35(1985), 163-174. MR812323 (87j:46101)

[2] L. Carleson, An interpolation problem for bounded analytic functions, Amer. J. Math. 80(1958), 921-930. MR0117349 (22:8129)

[3] J. Garnett, Bounded Analytic Functions, Academic Press, New York, 1981. MR628971 (83g:30037)

[4] P. Gorkin, K. Izuchi, and R. Mortini, Higher order hulls in $H^{\infty}$ II, J. Funct. Anal. 177(2000), 107-129. MR1789945 (2002e:46060)

[5] P. Gorkin and R. Mortini, Interpolating Blaschke products and factorization in Douglas algebras, Michigan Math. J. 38(1991), 147-160. MR.1091518(92b:46083)

[6] K. Hoffman, Banach Spaces of Analytic Functions, Prentice Hall, New Jersey, 1962. MR.0133008 (24:A2844)

[7] K. Hoffman, Bounded analytic functions and Gleason parts, Ann. of Math. 86(1967), 74-111. MR0215102(35:5945)

[8] K. J. Izuchi, Countably generated Douglas algebras, Trans. Amer. Math. Soc. 299(1987), 171-192. MR869406 (88b:46077)

[9] K. J. Izuchi, Factorization of Blaschke products, Michigan Math. J. 40(1993), 53-75. MR 1214055 (94d:30062)

[10] K. J. Izuchi, Interpolating Blaschke products and factorization theorems, J. London Math. Soc. (2) 50(1994), 547-567. MR1299457(95k:46086)

[11] K. J. Izuchi, The structure of the maximal ideal space of $H^{\infty}$, Sugaku Expositions 17(2004), 171-184. MR2095766

[12] K. J. Izuchi and Y. Izuchi, Factorization of Blaschke products and primary ideals in $H^{\infty}, \mathrm{J}$. Funct. Anal. 259(2010), 975-1013. MR2652179 (2011h:46070)

[13] K. J. Izuchi and Y. Izuchi, Factorization of Blaschke products and ideal theory in $H^{\infty}, \mathrm{J}$. Funct. Anal. 260(2011), 2086-2147. MR2756151

[14] V. Tolokonnikov, Blaschke products with the Carleson-Newman condition, and ideals of the algebra $H^{\infty}$, J. Soviet Math. 42(1988), 1603-1610. MR849297(88c:30041)

Department of Mathematics, Nimgata University, NiIgata 950-2181, Japan

E-mail address: izuchi@m.sc.niigata-u.ac.jp

AOYAMA-SHINMACHI 18-6-301, NiSHI-KU, NiIGATA 950-2006, JAPAN

E-mail address: yfd10198@nifty.com 